\title{
LA SEXUALIDAD Y LA FORMACIÓN DE MAESTROS: DE LOS SUJETOS, LA PEDAGOGÍA Y LAS PRÁCTICAS UNIVERSITARIAS
}

\section{SEXUALITY AND TEACHERS TRAINING: SUBJECTS, PEDAGOGY AND UNIVERSITY PRACTICES \\ Por: Paola Andrea Roa García1. Angélica Del Pilar Osorio G2, Eliath Forero3, Andrea Marcela Buitrago4}

\begin{tabular}{|l|}
\hline Recibido:01-04-2010 \\
\hline Aceptado:02-06-2010 \\
\hline
\end{tabular}

RESUMEN: Se exponen diferentes reflexiones alrededor del asunto de la Educación Sexual, la pedagogía, las prácticas universitarias en relación con la sexualidad y la formación de maestros, presentando un modo de asumir la investigación educativa, el investigador, la sexualidad y el ejercicio de maestro. Las reflexiones son resultado de la sistematización del IX conversatorio realizado por la Línea de Investigación Trayectos y Aconteceres: Estudios del Ser y el Quehacer del Maestro desde la pedagogía, donde se propone además el conversatorio como una experiencia pedagógica, reconfigurando así el sentido del "evento académico-investigativo" y se plantean diferentes problematizaciones acerca de lo educable o enseñable de la sexualidad, los saberes sobre la sexualidad y educación sexual y el posicionamiento de los sujetos de la educación sexual como tal.

PALABRAS CLAVE: sexualidad, educación sexual, formación de maestros, pedagogía.

SUMMARY: This paper exposes different reflections upon the Sexual Education subject, pedagogy, and university practices in relation to sexuality and the teachers formation, presenting a way to assume the educative research, researcher, sexuality and the teachers job. The reflections are the result of the IX symposium's memories presented by the researching line "Trayectos y Aconteceres: Estudios del Ser y el Quehacer del Maestro desde la pedagogía", where it's also proposed the symposium as a pedagogic experience, reconfiguring the sense of the "academic-researching event" and different problems about what it is teachable regarding to sexuality, the facts about it, sexual education and the positioning of the subjects on itare shown.

ZY WORDS: Sexuality, Sex education, teachers training, pedagogy.

\section{「TRODUCCIÓN}

te artículo presenta la reflexión realizada a partir de la sistematización del IX pnversatorio de Trayectos y Aconteceres: "LA SEXUALIDAD Y LA FORMACIÓN DE AESTROS: DE LOS SUJETOS, LA PEDAGOGÍA Y LAS PRÁCTICAS UNIVERSITARIAS" 
realizado el 27 de marzo de 2009. El conversatorio se constituye en una experiencia pedagógica pensada y organizada por la línea de investigación Trayectos y Aconteceres: Estudios del ser y el quehacer del maestro desde la pedagogía adscrita al Departamento de Biología de la Universidad Pedagógica Nacional, como experiencia se ha venido consolidando hace mas de 10 años con el propósito de posibilitar la reflexión de las prácticas y discursos relacionados con la escuela y los maestros a través de la puesta en escena de los discursos que circulan, esto a través de audiovisuales, expertos, y sobre todo con las voces de los sujetos maestros y estudiantes que se constituyen en los principales expertos alrededor de dichas prácticas.

El IX conversatorio emerge del devenir de la línea alrededor del objeto sexualidad y particularmente de la mirada que nos ha convocado los dos últimos años alrededor de los juegos de verdad relacionados con la sexualidad que emergen en la educación sexual, es así que surge la pregunta por los maestros y la formación de éstos en la Universidad, por tanto el conversatorio es planteado como uno de los escenarios de la pregunta por la educación sexual en las universidades y para el caso la Universidad Pedagógica como educadora de educadores, específicamente, en el Departamento de Biología, dado que es a los profesores y por tradición a los de biología que se les asigna hablar de sexualidad en la escuela; por tal razón resulta relevante preguntarse por las condiciones de posibilidad, los discursos y prácticas relacionadas con su "enseñanza", la postura pedagógica que les subyace, además de visibilizar ¿qué se dice allí?, ¿qué suscita en los profesores en formación?, ¿qué prácticas despliegan estos en la escuela y en la Universidad? ¿a qué lógicas obedecen estas prácticas y discursos?, visibilizando los saberes que posibilita y su relación con la constitución de sujetos.

Esta mirada de la educación sexual, se propone debido a la permanente interrogación de los "problemas" asignados a la sexualidad y no por el sujeto como experiencia singular (más allá del rol estudiante, maestro, adolescente, adulto, entre otros), por las múltiples estrategias (proyecto de educación sexual, educación para la vida y el amor, educación para la sexualidad y la formación ciudadana, los derechos sexuales y reproductivos) que pretenden generar la verdad acerca del sujeto, por la asignación de la sexualidad a la escuela desde una mirada educativa, masificadora y no desde la problematización de la enseñanza, los sujetos involucrados en ésta y la postura pedagógica que le subyace. Lo que sugiere retomar el asunto de la subjetivación en la formación de maestros.

De esta manera, se abren nuevas posibilidades de indagación no sólo en términos de las prácticas y discursos relacionados con la sexualidad que circulan en el medio universitario, sino alrededor de la escuela, el quehacer del maestro y la constitución de subjetividades, con el objeto de problematizar la formación de maestros y su relación con las prácticas escolares y los discursos que confluyen en ésta, como el de la sexualidad.

\section{EL CONVERSATORIO COMO EXPERIENCIA PEDAGÓGICA}


Un trayecto se define como el espacio a recorrer entre un lugar y otro, en esta misma medida, el acontecer hace alusión a la inmediatez por las características propias del instante que lo hacen único, y es justamente en la movilidad de los trayectos y en la inmediatez de los acontecimientos que se construyen significados, se consolidan prácticas, se escenifican discursos, se significan objetos, dicho en otras palabras se anuda la cultura.

Se puede visualizar entonces que tanto la idea de trayecto como la de acontecer remiten a la transformación constante, a un cambio en donde la misma sustancia puede adquirir varias formas dependiendo del contexto en que se escenifique. Desde ésta perspectiva se puede afirmar que tanto el objeto de estudio como las metodologías, e incluso los sujetos mismos que componen la línea están inmersos en un devenir constante, muestra de ello, es que los trayectos que se han recorrido durante la historia, han llevado no sólo a la exploración de nuevos caminos, sino también a la reflexión y consolidación de nuevas formas de recorrerlos, como se puede observar en el transcurrir que se describe a continuación:

Trayectos y Aconteceres, emerge del grupo de investigación Violencia y Escuela que venía trabajando con proyectos financiados por el Centro de Investigaciones de la Universidad CIUP desde el año 2000, luego, En el año 2005 el equipo se adentró en el estudio del embarazo adolescente; para el 2006, se trabaja sobre dos objetos de estudio: uno relacionado con la mirada hacia nosotros mismos indagando sobre la Enseñanza de la Biología en el Departamento de Biología de la Universidad Pedagógica Nacional, y otro, sobre la configuración y la historia de la sexualidad en la escuela, relacionada con lo encontrado en el proyecto sobre Madres Adolescentes Embarazadas llevado a cabo en el 2005; actualmente, la última de estas temáticas empieza a desbordarse dando paso hacia el delineamiento de nuevos horizontes desde los que se contempla la posibilidad de abordar el saber escolar, como temática de indagación.

Sin embargo y visto desde el trascender que demarca la historia de la línea, ésta sigue siendo Trayectos y Aconteceres, incluso, sin abandonar del todo su punto de emergencia, aquel instante inicial en el que se denominó Violencia y Escuela, es sólo que una y otra vez deviene en algo nuevo, que por ser nuevo no deja de hacer parte de lo anterior, se puede afirmar entonces que la línea sigue siendo Trayectos y aconteceres sólo que siempre con nuevos rostros, con nuevas formas, pero sobre todo con nuevas preguntas que incitan y avizoran otras transformaciones. En el caldo de cultivo de las transformaciones e inmersos en el devenir, surge el interrogante por cómo asir los acontecimientos de los que se pretende dar cuenta, se acude así a la metáfora de la urdimbre que más que una realización simbólica es un ejercicio de investigación desde el que se tejen y se destejen los hilos de la realidad que traspasan y conforman el objeto de estudio.

El investigador al igual que Penélope entreteje los hilos, anuda palabras, prácticas, objetos, discursos, establece puntos de interconexión entre el afuera y el adentro, pero también 
desteje, desanuda los hilos, para intentar dar cuenta de las relaciones que los imbrican. La interacción entre éste y la realidad es análoga a la que se da entre los hombres y las mujeres de las tribus de América del norte, ya que, mientras los primeros se ubican en la parte de adelante del lugar de reunión y se dan a la tarea de discutir sobre los asuntos de la comunidad, las segundas se hacen en la parte de atrás y se dedican a urdir, pero sus tejidos no se dan al margen de la reunión, sino que por el contrario cada una de las lazadas que interconectan un hilo con otro dan cuenta de las decisiones y desacuerdos que se ponen en escena durante la reunión.

El tejer se constituye así en una forma de escritura, que si se analiza no es ajena al investigador, quién también asiste a la realidad en procura de dar cuenta de ella, sólo que en el caso de éste, el ejercicio es dual, pues como ya se anotó, él además de seleccionar los hilos y anudarlos, se da a la tarea de destejer, de desenredar la maraña de interrelaciones que conforman la realidad, pues sólo de esta forma es posible dar cuenta de los acontecimientos, los cuales, asumen nuevas formas dependiendo del contexto en donde tengan lugar. Partiendo de esta perspectiva resulta pertinente pensar en la mirada arqueológica genealógica, planteada por Foucault, como una suerte de herramienta, de telar que posibilita entretejer los hilos que acuden a la conformación tanto de las interpretaciones como de los acontecimientos que se pretenden abordar. En el caso específico de los Juegos de verdad en la educación sexual, que es la temática que actualmente convoca al grupo, al ejercicio de urdir acuden como hilos conformantes: el saber, el poder, el sujeto, todos ellos inmersos dentro de las prácticas y las discursividades que se tejen alrededor de la sexualidad y de las posibilidades e imposibilidades de enseñar la misma.

Dichos hilos sin embargo, no se toman de madejas apartadas sino de una elaborada red de prácticas, discursos, imaginarios y objetos que Vásquez (2002) define como cultura, pero que en el caso del trabajo de la línea también es aplicable a la concepción de pedagogía que se consolida dentro del grupo y que se constituye en la urdimbre que constantemente se intenta destejer, decostruir para dar cuenta de las interrelaciones que tienen dentro de la misma y que posibilitan a los sujetos interrogarse desde el ejercicio de maestro.

Desde esta perspectiva se viene consolidando el conversatorio no sólo como un espacio de encuentro que se sitúa en el momento, que pasa y puede quedarse sólo en el instante que se olvida, el conversatorio se ha traducido en la posibilidad de incitar al conversar a la reflexión y a la pregunta; transcendiendo de esta manera la socialización de resultados de una investigación o el ejercicio unidireccional de escucha. El conversatorio, hace el llamado a la exposición de las ideas construidas y por construir, a la exposición de los sujetos, al ejercicio de la escritura de la creación y la recreación, es así una posibilidad de compartir con los otros, dando como resultado la configuración de nuevas preguntas y nuevos objetos, la ampliación de perspectivas, un momento para la reflexión. Una labor que se emprende como tarea colectiva, pero que afecta principalmente a los sujetos, donde 
se dan pasos en el devenir de los trayectos, al poner en juego no sólo los saberes sino la emocionalidad y el sentir; es por esto que proponemos el conversatorio como experiencia pedagógica entendiendo que una experiencia se caracteriza porque toca a los sujetos, hace que éstos se movilicen, los afecta a partir de las interrelaciones que se generan y del territorio donde se encuentran de modo que cuando unos sujetos están inmersos en una experiencia, ya no son los mismos que eran antes de involucrarse con ésta, cada una de estas experiencias impone un sello particular en sus vidas: éstas afectan directamente a los sujetos.

Para la ocasión ha sido la educación sexual el objeto de investigación que invita al pensamiento, a desmarañar la urdimbre y así han sido múltiples las formas en que cada participante ha decido aportar hilos, manos o agujas, estudiantes, maestros e investigadores que desde el telar de la pedagogía hacen que la confluencia de saberes y emociones posibiliten una forma de mirar diferente, eso que denominamos educación sexual, que nos permite pensar en la educación, en la escuela, en los niños y los jóvenes, en el acto pedagógico y sobre todo en las distintas formas de subjetividad.

\section{EL AUDIOVISUAL COMO CREACION E INCITACIÓN DE SABERES}

El audiovisual no es un recurso, ni una herramienta, así ha sido la forma en que la Línea desde hace un tiempo viene tejiendo hilos, construyendo otras miradas desde las miradas de otros, es con la edición, la música, las imágenes que se constituye saberes, saberes que buscan incitar a otros a la reflexión de ciertas prácticas y discursos. Con el audiovisual no se define la forma cómo o sobre qué pensar, sino se incita, generando problematización alrededor del mundo que nos rodea, haciendo otras lecturas de lo que somos, de aquello que nos constituye y de los sujetos de verdad que hemos configurado. Para el caso de la Educación Sexual los dos materiales construidos reescriben algunos aspectos del discurso que sobre la misma se ha podido visibilizar, agrupando algunos enunciados, como la salud sexual y reproductiva, la prevención, la promoción, los derechos sexuales y reproductivos, la condición de los embarazos jóvenes entre otros elementos que configuran algunas verdades sobre la sexualidad y la educación sexual.

Para constituir la urdimbre a la que hacemos mención, hemos logrado concluir que lo audiovisual es un campo de especial atención en cuanto a las posibilidades que ofrece, para entender esto es necesario recordar que como dice Jesús Martín Barbero (2003), estamos acostumbrados a confundir la comunicación con los medios y la educación con sus métodos, no se puede simplificar la acción del audiovisual a un uso técnico, definido simplemente por las posibilidades estéticas o de versatilidad para comunicar un mensaje; es sorteando esta confusión que el maestro posiciona su voz en el audiovisual desde la pedagogía como su campo. Esta claridad permite examinar las posibilidades de lo audiovisual para crear: para nosotros el audiovisual no es un recurso ni una herramienta, ha sido un lenguaje del cual nos hemos apropiado, apostamos por entenderlo como 
lenguaje audiovisual, y como tal puede ser la forma de propiciar otras miradas desde las miradas de otros.

La apuesta por lo audiovisual también se relaciona con cuestionar que si bien la tradición de la escritura ha tenido una especial relevancia en la academia, el poner lo audiovisual como lenguaje conlleva una complejidad en su estructura y uso, más allá del tradicional uso didáctico; el audiovisual es esa voz de maestro donde queremos hacer sentir nuestra creación, nuestra producción, y por tanto se hace apropiado para dar cuenta de la acción creadora del maestro y desde la pedagogía siendo usado para posibilitar y dar ocasión a mundos posibles. Pensar en las posibilidades del audiovisual como lenguaje, nos deja entender que con la edición, la música y las imágenes, se constituyen saberes; saberes que buscan incitar a otros a la reflexión de ciertas prácticas y discursos. Con el audiovisual, como lo dice Barbero construir el propio mundo y decirse a si mismo es posibilitar pensamiento, generar dudas e inquietar, es desubicar, traer a memoria para añadir otro valor a algo ya dicho, explotar la imagen desde la perspectiva del maestro, indagar por las opciones que deja el audiovisual para actuar desde un saber pedagógico.

Esta apuesta también implica que anclado a una reflexión de maestro, lo audiovisual no puede quedarse en dar fuerza a un discurso, o simplemente ser una forma de verdad o apoyar verdades con imágenes y sonidos; puede ubicarse en la acción creadora del maestro orientando este lenguaje, y es por esto que se asume desde la Línea de investigación como una forma efectiva de extender la acción creadora del maestro y posibilitar pensamiento. Bajo esta premisa el espacio del conversatorio ha sido la ocasión propicia para dar desarrollo a esta forma de entender y hacer audiovisual, pues desde los últimos años como parte del conversatorio, el audiovisual tomó un lugar importante como forma de dirigir y propiciar las discusiones; no como forma de transmisión de conocimientos acabados y verdades últimas, sino como elemento fundamental para problematizar el discurso que nos rodea y que pasa irreflexo, buscando una introspección con diversidad de lecturas de lo que somos, esas partes que nos constituyen, pero también las formas de sujeción a la verdad inventada y establecida.

De este modo mientras se articula un documento en imágenes y sonido, se propicia el cuestionarnos; esto cuando se hila con una intención, se enmarca en la crítica y la búsqueda de la posibilidad creativa no sólo desde la estética, pues si bien la reconoce y se apoya en ésta para dar un uso del lenguaje que permita constituir diversos significados, la característica fundamental de lo audiovisual está en su posibilidad discursiva, asumiendo que el audiovisual representa, antes que presentar una realidad y por tanto no es una captura inofensiva u objetiva.

\section{LOS EXPERTOS: De quienes conversan y lo que conversaron.}

La etimología de la palabra autoridad nos remite al sentido del auctor y el verbo augere sugiriendo la fuente o el origen de algo o la posibilidad de aumentar, agrandar o mejorar, será quizás este tipo de significaciones los que han permeado nuestra búsqueda de autoridad en casi todos nuestros planos de desenvolvimiento, exigimos y se nos exige 
autoridad como capacidad para controlar y ejercer influencia sobre otros, no es sino -para quienes somos maestros o estamos inmersos en la labor educativa- dar una mirada a los escenarios de los que hemos participado para notar que la preocupación por la autoridad hace parte en unos casos de los medios para educar y en otros de los fines para hacerlo; si bien, no es objeto de esta discusión, encontrar si es o no pertinente preocuparnos por la autoridad en el contexto educativo, si queremos mostrar cómo esta noción de la autoridad en quien habla sobre algo, es determinante, al parecer cuando se trata de un discurso académico. El estatuto de quien habla, diría Foucault (2005) comporta de criterios de competencia y de saber; instituciones, sistemas, normas pedagógicas, bien podría decirse que sobre medicina habla el médico, pues es quien tiene la autoridad para hacerlo. No obstante, el autor y la autoridad pueden ser vistas como puntos en los tejidos del discurso, de manera que el autor no es el "individuo que habla" sino el principio de agrupación del discurso como tal. Lo anterior nos permite hacer la pregunta entonces por ¿Quiénes son los autorizados para hablar de Educación Sexual? , ¿Quiénes son los expertos?. Si se tratara de encontrar "la verdad" de la Educación Sexual fácilmente encontraríamos que la autoridad para hablar estaría en el sitio del psicólogo, el médico o el sexólogo; pero si asumimos que el autor es una función y no un individuo, una función para desplegar, poner a circular los enunciados, cuestionar, afirmar, poner en el sitio de la crítica, entonces entendemos que es experto y autor quien ha construído una experiencia, que para el caso una experiencia de saber, que en relación al asunto que nos convoca la "educación sexual" está también en los maestros, en ejercicio y formación, en los miembros de diferentes comunidades educativas, estudiantes, directivos, orientadores; en quiénes se preguntan por cómo escribir al respecto, los grupos editoriales, en quiénes han construído proyectos y han emprendido indagaciones a muy distintos niveles, los investigadores y los asesores, en definitiva en quienes han transitado desde diversos lugares donde se han configurado los qués, los por qués y los cómos de la educación sexual, pero sobre todo las preguntas por lo que implica y los efectos que tiene educar para o en la sexualidad. Es por esto que no se asume al "experto" como quién tiene la verdad sobre sino como quien muestra el deseo de compartir sus saberes y sus experiencias con los otros, quien quiere poner en juego las verdades de las que tal vez es sujeto.

En este sentido, se asume a todos los asistentes al conversatorio como expertos, dado que se entiende la sexualidad como experiencia singular donde los trayectos de cada sujeto se constituyen en fuerzas de saber y poder que posibilitan acercarse a su problematización, de esta forma las disciplinas que tradicionalmente han representado la autoridad acerca de la sexualidad se constituyen solamente en otra voz, en otra experiencia alrededor de la misma, pues el objeto no es establecer consensos, ni nuevas teorías, ni mucho menos una didáctica de la sexualidad, sino y a través de la conversación como encuentro de fuerzas de distinta índole posibilitar visibilizar aquellas formas que adquiere el saber sobre la sexualidad, lo que incitan, lo que reprimen, y por supuesto cuestionarlo desde el saber propio de los maestros la pedagogía, es así que surge el cuestionamiento sobre la enseñanza y en ese sentido la denominada educación sexual. 
Decimos fuerzas de distinta índole porque las personas que constituyeron el conversatorio provienen de contextos distintos, profesores de las Normales Superiores, de instituciones educativas (secundaria y universidad) públicas y privadas, estudiantes de diferentes licenciaturas de la UPN, asesores de Bienestar Universitario, entre otros, y son contextos distintos no solo por su lugar actual de desempeño, desde la perspectiva del grupo, se propone enunciar el contexto como las condiciones de posibilidad de las relaciones que emergen, dicha noción está enmarcada en la idea del contexto como un escenario que permea las construcciones de significado que tienen lugar en el mismo. Al pensar en las condiciones de posibilidad, se está reconociendo que ni la educación ni el docente están "in vacuo" sino que por el contrario tanto las prácticas como los discursos que desde allí se tejen están enmarcadas en escenarios reales, que más allá de constituirse como cronotopos de posibilidad impactan en la forma que se le da a la educación misma.

De allí que se pueda afirmar que los contextos son singulares históricamente, que las problemáticas varían con la historia (tiempo y espacio) y los lugares, planteando siempre nuevas preguntas alrededor de las formas de enunciación, de visibilidad de los saberes, las fuerzas que los instituyen o los invisibilizan y su relación con los sujetos. De este modo a partir del audiovisual como fuerza de incitación se desarrolla el conversatorio, a través de la tematización realizada del mismo a partir de la transcripción del registro en audio y escrito, se encuentra como temáticas que hacen su aparición regularmente las siguientes:

El conocimiento sobre el sexo. Este hace referencia a los modos en que se visibiliza la sexualidad como objeto de educación, es así que aquello que se muestra es el sexo y éste entendido como la fisiología de los aparatos reproductores, así como los métodos de planificación. Llama la atención que se interroga por qué al hablar de sexualidad terminamos hablando de sexo y éste desde la genitalidad, pero al mismo tiempo tal genitalidad quiere ser extraída de lo que sería sexualidad y para el caso educación sexual, y ello porque se asume que en la actualidad debido a todas las fuentes de información a las que se tiene acceso, no es necesario hablar de esto. En este sentido el conocimiento alrededor de la sexualidad toma un modo de ser diferente, ya no es el de la información, sino como se constituyen sujetos desde ella, y es así que cobra fuerza la idea de ser humano que permea toda la conversación realizada.

El error de la educación sexual: El embarazo. Aparece regularmente que uno de los problemas de la educación sexual es el embarazo, bajo ello se asume adolescente como aquel que está en la escuela secundaria, y adulto al que está fuera de ella aunque tenga la misma edad, en ese sentido es que se constituye en un error de la escuela, pues es el sistema educativo quien falla en la potencial producción del recurso humano que tiene a su cargo. Dado que se enuncia que la adolescente embarazada no tendrá posibilidad de estudiar, de constituir un proyecto de vida. Sin embargo, al enunciar que las abuelas fueron madres a los 13 años, surge un silencio que posibilita interrogar cómo es que hemos 
llegado a pensar que el embarazo es un error, lo cual sugiere que los maestros podemos interrogar la educación desde la historia cultural y hacer otras lecturas que a través de la práctica pedagógica pueden posibilitar la constitución de otras subjetividades.

De la certeza y la generalización. La ciencia del sexo: la sicología y la medicina. Surge entonces la pregunta en cómo no quedarnos en polarizaciones, ni en los extremos de la particularidad pero tampoco en los de la universalidad y esto porque se interroga si le sexualidad debe ser enseñada en todos los contextos, pero también que tal enseñanza cambia de acuerdo a los mismos, así la noción de derechos sexuales y reproductivos que emerge hace poco tiempo y que se visibiliza en la estrategia educación para la sexualidad y la ciudadanía, y que se concreta en las mesas de discusión del plan decenal, aparece como una generalización que invisibiliza a los sujetos, así como también hace de la sexualidad un problema donde los expertos en sicología y medicina, la resuelven desde teorías universales que descartan las experiencias singulares y los saberes que se construyen a partir de ellas. De este modo, se comprende porque los proyectos transversales en las instituciones educativas se quedan en documentos extensos de poca práctica, aunque la práctica de la sexualidad se hace todo el tiempo.

La educación sexual como problema multidisciplinario. Entonces se reafirma que el abordaje de la sexualidad en la escuela es un problema multidisciplinario, es decir que debe trabajarse desde todas las disciplinas, es allí donde implícito esta el asunto de la verdad y cómo se constituye, es decir el juego que se impone desde los saberes que deben enseñarse porque han sido validados desde las disciplinas científicas, y lo que desde ellos los sujetos deben pensar y hacer sobre sí mismos, es de este modo como la sexualidad en la escuela a través de su educación y acompañada por las fuerzas que confluyen de otros escenarios queda referida a la genitalidad, a la salud, la prevención, el desarrollo integral, donde es el embarazo, el SIDA y las ITS los problemas que deben resolverse desde la información y su educabilidad. Se enuncia entonces que el error de la escuela es que no ha logrado articular las disciplinas para abordar la sexualidad y su enseñanza, de allí se desprende que tal vez el asunto de la sexualidad no es cuestión solamente de las disciplinas y que además no debe ser abordado como problema, sino problematizarlo para interrogarnos sobre cómo construimos las certezas de aquello que somos o que nos enseñaron y aprendimos que debemos ser.

La sexualidad es innata a la práctica pedagógica. Así, el maestro es solo un ejecutor de los planes expuestos por las políticas relacionadas con la educación de la sexualidad, es entonces que los profesores somos guías, garantes de los derechos sexuales, de este modo surge el cuestionamiento por la práctica pedagógica, el estatus del maestro en la actualidad como funcionario y su saber propio, emerge entonces la disputa entre las disciplinas y la pedagogía, no obstante la pedagogía queda reducida a un ejercicio de instrucción, así se válida el profesor como servidor, aunque se establece la pregunta por la intelectualidad del maestro y su devenir histórico. De allí se cambia la mirada hacia al 
maestro, y la conversación resulta en visibilizar su ser singular, su historicidad, y se enuncia que la sexualidad somos los sujetos mismos, de ese modo no hay un currículo, ni una evaluación alrededor de la sexualidad, ésta se educa en las prácticas mismas, en las experiencias que a diario se entrecruzan, de esta forma la sexualidad se configura en un pretexto que muestra la necesidad de la pregunta por los sujetos, y emerge de nuevo la noción de ser humano, al parecer éste se constituye en su educación, de este modo se visibliza la idea de enseñar lo correcto alrededor de la sexualidad y se habla del potencial formativo del hombre y que se concreta en la escuela. Podría entonces pensarse que la pregunta por el sujeto, está asociada a los nuevos modos de ser del conocimiento en la actualidad, ya no es la información, sino la mercantilización de experiencias de vida, de estilos de vida, es posible que desde los derechos sexuales y reproductivos se validen ciertas formas y experiencias de vida. Así, si la sexualidad es innata a la práctica pedagógica y se constituye en experiencia, su educabilidad no ha sido siempre la misma, ni siempre ha sido necesario educar en sexualidad.

\section{LA EDUCACIÓN SEXUAL EN LA UPN. Los asesores, los profesores, los estudiantes sujetos de la educación sexual.}

En la segunda parte del conversatorio se realiza un panel con los que se llamarían expertos desde ciertos lugares comunes, expertos en la medida que han realizado estudios de pregrado y posgrado alrededor de las disciplinas que se constituyen en autoridad para hablar de sexualidad, sin embargo y de forma intencional se incluyen en el panel tres maestras, la primera que desde hace mas 15 años ha enseñado educación sexual en la universidad, la segunda que desde la genealogía ha interrogado la educación sexual en la UPN y la tercera estudiante de pregrado que desde su experiencia posibilita interrogar lo que los otros panelistas exponen. A continuación se exponen las nociones más relevantes en sus exposiciones.

La sexualidad como un problema socialmente relevante: El propósito es el bienestar humano. A través de la prevención integral, se busca la promoción de factores protectores, intervención sobre riesgos y determinantes del problema, el mejoramiento de la calidad de vida y el desarrollo humano, mediante la comprensión y acción transformadora de las condiciones y factores que los debilitan, por eso deben fundamentarse en concepciones claras de ser humano, maestro, educando, comunidad educativa, pedagogía, escuela, promoción y prevención, que apunten a la transformación de la cultura institucional y sus interacciones. De este modo se propone trabajar sobre los sueños, porque la sexualidad es solo un pretexto para interrogar los seres humanos, por esto debemos volvernos expertos en seres humanos y no como lo hizo la escuela y el maestro que se convirtieron en expertos en disciplinas y perdieron la noción de lo humano (Parra, 2009).

Saberes y prácticas de la educación sexual: Una experiencia compartida. La cátedra de educación sexual pretendía acercarse a la comprensión de la sexualidad desde los diversos discursos y prácticas que en lo biológico, lo erótico, lo afectivo, lo social y lo educativo 
han construido consciente o inconscientemente en nuestras vidas lo que llamamos "sexualidad humana". Referentes anatómicos y neurofisiológicos desde la "erudición científica" permitían redescubrían la propia anatomía y neurofisiología sexual, enfrentada a la carga cultural que pesa sobre las expresiones de sexo, genitalidad, erotismo y sexualidad en cada historia de vida. Una mirada a la historia de la sexualidad, las diferentes civilizaciones y culturas, caminando de la mano de las más importantes expresiones: desde el mundo de nuestras culturas indígenas, la expresión griega y romana, nuestra herencia judeo- cristiana hasta llegar a nuestras expresiones actuales. Se buscaba entonces la deconstrucción/reconstrucción de sus representaciones sociales sobre la sexualidad, como camino de búsqueda del sentido personal de la sexualidad, un recorrido más conscientes de las violencias de las que formamos parte, de los sistemas de creencias, pensamientos y lenguaje cotidiano que como fenómeno social y personal, con el fin de permitirnos asumir con mayor conciencia nuestras posturas, concepciones y prácticas discursivas sobre la sexualidad (Fernández, 2009).

Lo enunciable y lo visible de la sexualidad. Los discursos sobre sexualidad que circulan en el ámbito universitario están asociados generalmente con la genitalidad, el embarazo en madres estudiantes del nivel superior, la configuración de roles, las enfermedades de transmisión sexual y lo fisiológico, se evidencia una multiplicidad de tendencias en relación con la forma de asumir la identidad. Dentro de la diversidad universitaria es claro que no se valida un solo discurso, aquí no hay certezas, cada uno tiene la opción de elegir, es por esto que en este lugar la sexualidad también toma la forma de chistes, metáforas o historias. Atendiendo a los discursos que circulan sobre sexualidad es fundamental que se asuma una mirada crítica desde la problematización de cómo es asumida la sexualidad, por lo que se hace una invitación para que desde las prácticas universitarias y la pedagogía logre movilizarse un pensamiento más dado hacia la reflexión que permita generar una mirada diferente. (Quente, Sánchez \& Bernal. 2009)

La investigación de la práctica pedagógica. La mirada genealógica de los maestros de la U a la cátedra de educación sexual. La cátedra de educación sexual en el Departamento de Biología emerge en 1991 relacionada con los problemas de salud donde se incluyen los comportamientos sexuales de interés desde inicios de los 80 , cobra vigencia desde los discursos relacionados con la salud -de una amplia trayectoria desde los 50 relacionado a la estrategia del desarrollo-, donde los puntos de encuentro con la disciplina biológica la validan en el programa de formación, además, el rol desempeñado por el maestro, dadas las dinámicas de la escuela y la responsabilidad que se le otorga como trasmisora de los valores culturales y los derechos - entre ellos la salud y el bienestar-, en la actualidad, desde la misión de la Universidad, le dan pertinencia y vigencia, más aún cuando el discurso se relaciona con la salud, el bienestar, el derecho, la seguridad y la vida. Así, la cátedra de educación sexual se constituye en una estrategia que despliega ciertas formas de saber en relación a los sujetos, a los maestros, a la escuela, pero también es fuerza en la medida que incita dichos saberes y reprime otros a través de prácticas y discursos específicos que convergen en la necesidad de educar en sexualidad desde la perspectiva de 
salud. Es importante, entonces señalar que los discursos y prácticas relacionados con la sexualidad no han sido siempre los mismos, dado que las fuerzas y formas que los visibilizan, les otorgan permanencia o ausencia, son dinámicas, y varían a través del tiempo intensificándose o haciéndose invisibles; es así que en los 80 se habla de educación para la salud con el ánimo de mantener el bienestar y el desarrollo, a finales de ésta década el discurso del desarrollo se traslada al sujeto, lo que posibilita hablar de desarrollo humano y ante el supuesto incremento de enfermedades trasmisibles y el embarazo adolescente, los comportamientos sexuales antes involucrados en educación para la salud, se consolidan en la llamada educación sexual, es así que, a mediados de los 90 se hace necesaria la educabilidad de la sexualidad, que ante su inminente fracaso -debido al incremento de enfermedades, violencia, carencia de valores- a principios de los 2000 desde la noción de prevención y promoción se integra a la formación ciudadana, solapada con el discurso de derecho y la línea de fuerza mantenida durante los últimos 30 años la salud escolar, que en la actualidad, se justifica desde los discursos de calidad de vida, seguridad, entre otros (Roa, P. 2008).

\section{LA MARAÑA: ALGUNOS HILOS POR DESANUDAR}

Los hilos que entretejen los fenómenos y los acontecimientos, no siempre se pueden desanudar de manera fácil, de hecho la realidad, muchas veces se presenta ante los ojos del investigador como una maraña en donde es difícil determinar cuáles y en qué momentos específicos suceden las imbricaciones entre un hilo y otro, en el caso de la educación sexual, hay algunas marañas que aún no se han desanudado del todo, las cuales adquieren formas de interrogantes y problematizaciones que entran a nutrir las reflexiones que tienen lugar dentro de la línea.

En el marco del conversatorio se hicieron evidentes las marañas relacionadas con los siguientes interrogantes: ¿se puede hablar de uno o de varios juegos de verdad en la educación sexual?, ¿la sexualidad es una cátedra que se puede enseñar?, ¿cómo se incluyen el placer y el cuerpo en la enseñanza de la sexualidad?. Dichos interrogantes están atravesados, todos, por un conjunto de interrelaciones mediadas por el poder, el saber y la subjetividad, de ahí que sea necesario atender a éstos no como hechos o problematizaciones aisladas sino como un entretejido en el que todos los componentes son partes importantes, en la medida en que entran a dar luces, cabe anotar además que las respuestas no siempre son unívocas, sino que por el contrario pueden adquirir muchas formas dependiendo de la panorámica y el ángulo por donde se miren.

En el caso del juego o los juegos de verdad en la sexualidad, por ejemplo, para llegar a determinar si la categoría es plural o singular se hace necesario analizar hasta que punto todas las jugadas que se dan en torno a la educación sexual están mediadas por las mismas reglas constitutivas y lo que cambia son los sujetos y las formas de actualizar dichas reglas, es decir, si las variaciones son simplemente de carácter regulativo y se mantiene la esencia misma del juego, o si por el contrario las actuaciones y enunciaciones que se construyen en 
torno a la misma varían de manera sustancial afectando incluso el plano de los constitutivo dando así origen a diferentes juegos.

Desde ésta perspectiva se hace pertinente seguir desanudando una y otra vez los hilos para entrar a dar cuenta de las múltiples respuestas que se pueden entretejer en torno a los interrogantes antes suscitados, de ahí la importancia que se le da al conversatorio, ya que, desde éste es posible avisorar otros hilos constitutivos, desanudar nuevas marañas e incluso destejer sobre urdimbres ya hiladas.

\section{BIBLIOGRAFIA}

Brea, J. (2007). Cultura _ RAM. Mutaciones de la cultura en la era de su distribución electrónica. CIBERCULTURA. Barcelona: Editorial Gedisa.

Martín - Barbero, J. (2003), La educación desde la Comunicación. Enciclopedia Latinoamericana de Sociocultura y Comunicación. Bogotá: Editorial Norma.

Canguilhem, G. (1966). Lo Normal y lo Patológico. México: SIGLO XXI.

Corrales, R. Giraldo V. La Sexualidad En Los Jóvenes Un mundo disonante, problemático y conflictivo. En Línea: http://tone.udea.edu.co/revista/sep96/sexujove.html\#nota Fecha de revisión: Marzo 7 de 2005.

Faur, E. (2006)“Género, Masculinidades y Políticas Reconciliación Familia - Trabajo” En: Revista Nómadas (№ 24 Abril 2006). Bogotá: Universidad Central. Departamento de investigaciones DIUC.

Fernández, M. (2009) Saberes y prácticas de la educación sexual: Una experiencia compartida

Focault, M. (1982). Hermenéutica del Sujeto. Argentina: Altamira.

Focault, M (1999). Ética del cuidado de sí como práctica de la libertad. En: Estética, Ética y hermenéutica. Editorial Paidós.

Focault, M. (2002) Historia de la Sexualidad. La Voluntad del Saber. Argentina: Siglo XXI Editores.

Focault, M. (2005) Historia de la sexualidad 2 El uso de los placeres. 5 Edición. México: Siglo XXI Editores

Focault, M (1999) "Sexualidad y poder: Conferencia en la Universidad de Tokio, el 20 de abril de 1978". Obras Esenciales. Vol. III. Estética, Ética y Hermenéutica. España: Paidos. Focault, M. (1990) Tecnologías del Yo y Otros Textos Afines. España: Paidos.

Larrosa, J. (1995). Escuela, Poder y Subjetivación. Madrid: La piqueta.

Martínez, A. (2004). De la escuela expansiva a la escuela competitiva, dos modos de modernización en América Latina. Barcelona: Anthropos.

Ministerio de Educación Nacional. Proyecto Nacional de Educación Sexual. (1994) Colombia.

Moreno, A. "Ciudadanía y Sexualidad, En La Ciudad De Buenos Aires" (2006). En: Revista Nómadas (№ 24 abril 2006). Bogotá: Universidad Central. Departamento de investigaciones DIUC. 
Múnera, A.(2006) "La sexualidad desde la iglesia católica" En: Revista Javeriana. Enerofebrero 2006. Bogotá.

Muñoz. D. "Sexualidades Ilegitimas. Biopolítica Heterosexista y Política De Reconocimiento" (2006). En: Derechos, cuerpo y sexualidad Revista Nómadas (№ 24 abril 2006). Bogotá: Universidad Central. Departamento de investigaciones DIUC.

Nietzsche, F. (1972) La genealogía de la moral. Madrid: Alianza.

Noguera, C. (2003) Medicina y Política. Discurso médico y prácticas higiénicas durante la primera mitad del siglo XX en Colombia. Medellín: Fondo Editorial Universidad EAFIT.

Ospina, A. Lara, L. (2002) Representaciones sociales en un grupo de adolescentes en torno a factores de riesgo en su salud sexual, estudio realizado en una institución oficial. Trabajo de grado para optar al título de Licenciado en Biología. Universidad Pedagógica Nacional. Parada, J. (2005). El embarazo adolescente le cuesta al país. Unimedios. Periódico de la Universidad Nacional de Colombia.

Pardo, U. (1991) Un estudio de embarazo en adolescentes en 11 instituciones colombianas. Revista Colombiana de Obstetricia y Ginecología.

Parra, Luis. (2009). El educador, la promoción y la prevención integral.

Pedraza, Z. (2004) “Intervenciones estéticas del Yo: Sobre Estético-Política, Subjetividad y Corporalidad" En: Debates Sobre el Sujeto. Perspectivas Contemporáneas. Bogotá: Siglo del hombre editores.

Pedraza, Z. (2001). "Sentidos, movimiento y cultivo del cuerpo: política higiénica para la nación" En: Herrera M, Díaz C. Educación y cultura política una mirada multidisciplinaria. Bogotá: Universidad Pedagógica Nacional.

Plan decenal de infancia para Colombia. Objetivos, Metas y estrategias de Plan de País. Documento en borrador para discutir. (2004 - 2015).

Puleo, A. (1992). Dialéctica de la sexualidad, género y sexo en la filosofía contemporánea. Madrid: Cátedra.

Quente A, Sánchez, Bernal L. (2009) Lo enunciable y lo visible de la sexualidad. Documento de Trabajo. Universidad Pedagógica Nacional.

Roa, P. et al. (2005). Violencia y Escuela: Representaciones de adolescentes embarazadas y/o madres acerca del embarazo, del ser madres y de asumir la maternidad de instituciones educativas de Bogotá. Informe de Investigación. CIUP. UPN. Bogotá.

Roa, P. et al. (2006). Trayectos y Aconteceres: Análisis de las prácticas discursivas acerca de la sexualidad en la escuela colombiana entre 1950 y 1960. Informe de Investigación. CIUP. UPN. Bogotá.

Roa, P. et al. (2008). Trayectos y Aconteceres: La educación sexual en Colombia: análisis de los juegos de verdad en la escuela 1990-2007. Informe de Investigación. CIUP. UPN. Bogotá.

Roa, P. (2008). Las prácticas discursivas sobre sexualidad a través de la emergencia de la cátedra de educación sexual en el Departamento de Biología de la UPN. Trabajo de Grado. Especialización en Pedagogía. Facultad de Educación. UPN.

Silveira, S. La dimensión de género y sus implicaciones en la relación entre juventud, formación y trabajo. CINTERFOR-OIT, Uruguay. Organización Internacional del Trabajo: 
Jóvenes, formación

empleo.En:http://www.ilo.org/public/spanish/region/ampro/cinterfor/temas/youth/evento s/mex/ibero/resumen/mesa_v/sara/index.htm

Torres, R. (1953). Inspector Central de las escuelas del magisterio de la iglesia. Problemas de Educación. El sistema educativo de Don Bosco en las pedagogías general y especiales. Madrid.

USECHE, B. (1993) La educación Sexual en los jóvenes. En: Revista educación y cultura N 32. FECODE . Bogotá.

Vargas N. Adolescentes Embarazadas en Costa Rica: Voces de Esperanza. Instituto Nacional de la Mujer. Costa Rica. INAMU. www.casapres.geo.cr Fecha de Consulta: Junio 10 de 2005.

Velásquez, C. (2001). El problema de la constitución de sujeto: una mirada desde la experiencia de sí a partir de la sexualidad en un grupo de jóvenes raperos de la localidad de Bosa en Bogotá. Tesis maestría psicología comunitaria Pontificia universidad Javeriana. Bogotá.

Zuluaga, O. (1999) Pedagogía e Historia: La historicidad de la pedagogía. La enseñanza un objeto de saber. Antioquia: Anthropos siglo del Hombre editores.

Zuluaga, O. Et al. (2003) Pedagogía y epistemología. Colección Pedagogía e Historia. Grupo Historia de la Práctica Pedagógica. Colombia: Magisterio. 